Mostafa Fazly* and Henrik Shahgholian

\title{
Monotonicity formulas for coupled elliptic gradient systems with applications
}

https://doi.org/10.1515/anona-2020-0010

Received October 17, 2018; accepted January 6, 2019.

Abstract: Consider the following coupled elliptic system of equations

$$
(-\Delta)^{s} u_{i}=\left(u_{1}^{2}+\cdots+u_{m}^{2}\right)^{\frac{p-1}{2}} u_{i} \text { in } \mathbb{R}^{n},
$$

where $0<s \leq 2, p>1, m \geq 1, u=\left(u_{i}\right)_{i=1}^{m}$ and $u_{i}: \mathbb{R}^{n} \rightarrow \mathbb{R}$. The qualitative behavior of solutions of the above system has been studied from various perspectives in the literature including the free boundary problems and the classification of solutions. For the case of local scalar equation, that is when $m=1$ and $s=1$, Gidas and Spruck in [26] and later Caffarelli, Gidas and Spruck in [6] provided the classification of solutions for Sobolev sub-critical and critical exponents. More recently, for the case of local system of equations that is when $m \geq 1$ and $s=1$ a similar classification result is given by Druet, Hebey and Vétois in [17] and references therein. In this paper, we derive monotonicity formulae for entire solutions of the above local, when $s=1,2$, and nonlocal, when $0<s<1$ and $1<s<2$, system. These monotonicity formulae are of great interests due to the fact that a counterpart of the celebrated monotonicity formula of Alt-Caffarelli-Friedman [1] seems to be challenging to derive for system of equations. Then, we apply these formulae to give a classification of finite Morse index solutions. In the end, we provide an open problem in regards to monotonicity formulae for Lane-Emden systems.

Keywords: Coupled elliptic systems, monotonicity formulae, homogeneous solutions, stable solutions, fractional Laplacian operator

MSC: 35J60, 35J50, 35B35, 35B45

\section{Introduction}

\subsection{Background}

Our objective in this paper is to establish monotonicity formulae for solutions of the following coupled elliptic system of equations

$$
(-\Delta)^{s} u_{i}=|u|^{p-1} u_{i} \text { in } \mathbb{R}^{n}
$$

and classify finite Morse index solutions when $0<s \leq 2, p>1, m \geq 1, u=\left(u_{i}\right)_{i=1}^{m}$ and $u_{i}: \mathbb{R}^{n} \rightarrow \mathbb{R}$. Note that when $m=1$ the above is known as the Lane-Emden equation. An important feature of system (1.1) is the variational structure of the coupled equations of the form

$$
-\Delta u=\nabla H(u) \text { in } \mathbb{R}^{n} .
$$

Note that (1.1) is a particular case of (4.33) for $H(u)=\frac{1}{p+1}|u|^{p+1}$. Due to this variational structure, qualitative behaviour of solutions of system (4.33) has been studied extensively in the context of elliptic partial differen-

\footnotetext{
*Corresponding Author: Mostafa Fazly, Department of Mathematics, The University of Texas at San Antonio, San Antonio, TX 78249, USA, E-mail: mostafa.fazly@utsa.edu

Henrik Shahgholian, Department of Mathematics, KTH, Lindstedtsvägen 25, 10044 Stockholm, Sweden,

E-mail: henriksh@math.kth.se
} 
tial equations from both pure and applied mathematics perspectives. Let us mention that Andersson et al. in [4] considered system (1.1), up to a negative sign, when $p=0$ and $s=1$ which reads

$$
\Delta u_{i}=\frac{u_{i}}{|u|} \chi_{\{|u|>0\}}
$$

This is closely related to minimizers of the energy

$$
\int\left(\sum_{i=1}^{m}\left|\nabla u_{i}\right|^{2}+2|u|\right) d x
$$

and they studied the regularity of free boundaries. To prove regularity results in [4], they established a monotonicity formula that is inspired by the one given by Weiss in [44, 45]. We also refer interested readers to $[3,7]$ for regularity results on cooperative systems and to the book of Petrosyan, Shahgholian and Uraltseva in [36] for more information. It should be remarked that as a particular case, one can consider the case of two components, that is $m=2$, and $p=3$ namely

$$
\left\{\begin{aligned}
(-\Delta)^{s} u & =\left(u^{2}+v^{2}\right) u \text { in } \mathbb{R}^{n}, \\
(-\Delta)^{s} v & =\left(u^{2}+v^{2}\right) v \text { in } \mathbb{R}^{n}
\end{aligned}\right.
$$

Note that the above system is a special case of the nonlinear Schrödinger system that is

$$
\left\{\begin{array}{l}
(-\Delta)^{s} u=\left(\mu_{1} u^{2}+\beta v^{2}\right) u \text { in } \mathbb{R}^{n}, \\
(-\Delta)^{s} v=\left(\mu_{2} u^{2}+\beta v^{2}\right) v \text { in } \mathbb{R}^{n},
\end{array}\right.
$$

where $\mu_{1}, \mu_{2}, \beta$ are constants. We would like to mention that most of the results in the paper are valid for (1.6) as well. We refer interested readers to $[34,41,42]$ and references therein for more information regarding classification of solutions of system (1.6) for various parameters $\mu_{1}, \mu_{2}$ and $\beta$.

\subsection{Tools and methodology (Historical development)}

Semilinear elliptic equations (with almost four decades of history) are probably the most widely and intensely studied equations in PDE. The simplest equation in this class is expressed as

$$
\Delta u=f(u),
$$

and exhibits as many possible features, as the right hand side nonlinearity $f(u)$ may do. This equation has also been studied from so many different perspectives, that any attempt to try to list them here would probably fail. There are however two different types of questions, of interest to the current authors, that seem to be interconnected and developed in parallel, but almost separately:

i) Analysis of the singular set: $\{x: \nabla u=0\}$.

ii) Stability and Liouville's type questions.

One can actually add a third less studied, and probably more complicated problem to the above, which is:

iii) Structure of the blow-up set: $\{x:|u(x)|=\infty\}$.

In studying the above problems, experts have (in many cases) developed parallel tools and ideas to handle technical passages of the analysis of questions in each cases. Two of these tools, also utilized in this paper, are Monotonicity functional, and Blow-up/down Analysis.

The particular monotonicity functional used here combines the energy functional along with a balanced term (see below for explicit form). The use of monotonicity functional (in the way we present here) can be traced back ${ }^{1}$ to the work of Fleming in [25] for area minimizing currents which has subsequently been proved

1 It is worth mentioning that a different type of almost monotonicity functional was used by Arne Beurling in his thesis: A. Beurling, Etudes sur un probleme de majoration, thesis, (Uppsala), 1933. The monotonicity functional of A. Beurling states 
by Allard in [2] for stationary rectifiable $n$-varifolds, that was later developed further by others. The monotonicity functional that we shall use in this paper originates in the study of harmonic maps by Price in [37] and Schoen and Uhlenbeck in [40], see also [39]. And in connection to free boundary problems, it was first used by $\mathrm{Ou}$ [5], and developed later by Weiss in [45] and others in various forms. In the context of semilinear heat equations, a similar monotonicity formula is given by Giga and Kohn in [28] and for the corresponding elliptic equation by Pacard [35].

The second main tool, blow-up/down analysis, has its origin in local regularity theory and the so-called linearization technique (also called harmonic blow-up). To study local structure of level surfaces of a solution to the above semilinear problem, one considers scaled version of the problem and classifies the limit manifold of such scalings. The limit manifold naturally carries information of the local behavior of the solutions and hence one can then with some devices link back this behavior to the local problem and deduce the expected properties. It is now that the role of monotonicity functional become crucial and indispensable in that one can use the fact that when blowing up a solution, the monotonicity functional, call it $E(r, u)$, being monotone will have a limit. At the same time this functional has a nice scaling properties $E(r s, u)=E\left(s, u_{r}\right)$ for $r, s>0$ and $u_{r}$ a correctly scaled version of $u$. Hence one obtains

$$
E\left(0^{+}, u\right)=\lim _{r \rightarrow 0} E(r s, u)=\lim _{r \rightarrow 0} E\left(s, u_{r}\right)=E\left(s, u_{0}\right),
$$

so that $E\left(s, u_{0}\right)$ is constant. Now a strong version of the monotonicity functional asserts that the only time $E$ is constant is when the function $u_{0}$ is homogeneous, where the order of homogeneity is dictated by $E$. Since the same can be done by blowing-down, we will have that $u_{0}, u_{\infty}$ are homogeneous of same order. Next if one can prove that the homogenous solutions are unique we must then have $u_{0}=u_{\infty}$ and hence $E\left(0^{+}, u\right)=E(\infty, u)$, and we arrive at $E(r, u)=$ constant. Therefore $u$ is itself homogeneous, and already classified.

In the regularity theory of level surfaces the classification of homogeneous global solutions is one of the key elements of the theory, and many time a very hard nut to crack, in higher dimensions; in two dimensions homogenous solutions can be computed directly. In the stability theory and Liouville type problems this part is based on computations of eigenvalues of the corresponding Laplace-Beltrami on the sphere, as also done in this paper. As shown here below (and in many preceding papers by experts in this area) the only homogenous solutions in the appropriate space are the trivial solutions. In particular, the energy functional becomes zero and hence $E(r, u)=0$. From here one deduces that $u \equiv 0$ is the only solution satisfying the given condition.

It is worthwhile remarking the fundamental feature of these problems, that in some cases depend on the dimension and order of homogeneity are directly a consequence of the eigenvalues of Laplace-Beltrami, where in the case of index-theory results in the computation of eigenvalues for nonlinear Laplace-Beltrami, that in turn implies that non-trivial solutions do not exists in certain range of values. These ideas have their origin in the regularity theory of minimal surfaces, that boils down to proving minimal cones do not exists in dimensions less than eight.

\subsection{Problem setting}

Our main technique is to derive monotonicity formulae for solutions of (1.1) for various values of parameter $0<s \leq 2$. To provide such monotonicity formulae we consider various cases.

Case $s=1$. Consider the following functional for every $\lambda>0$ and $x_{0} \in \mathbb{R}^{n}$

$$
E_{1}\left(u, \lambda, x_{0}\right):=\lambda^{-n+2 \frac{p+1}{p-1}} \int_{B_{\lambda}\left(x_{0}\right)}\left(\frac{1}{2} \sum_{i=1}^{m}\left|\nabla u_{i}\right|^{2}-\frac{1}{p+1}|u|^{p+1}\right)+\frac{1}{p-1} \lambda^{-n+2 \frac{p+1}{p-1}-1} \int_{\partial B_{\lambda}\left(x_{0}\right)}|u|^{2},
$$

then the following monotonicity formula holds for classical solutions of (1.1).

that for a jordan curve $y$, the product of the harmonic measures for both sides of the curve can be controlled as follows: $\omega_{1}\left(B_{r}(z) \cap y\right) \cdot \omega_{2}\left(B_{r}(z) \cap y\right) \leq A r^{2}$, where $z \in y$, and $\omega_{1}, \omega_{2}$ denote the harmonic measures on each side of the curve. 
Theorem 1.1. Suppose that $u$ is a solution of (1.1) for $s=1$. Then, $E_{1}$ is a nondecreasing function of $\lambda$ and in fact

$$
\frac{d E_{1}}{d \lambda}=\lambda^{-n+1+2 \frac{p+1}{p-1}} \int_{\partial B_{\lambda}\left(x_{0}\right)} \sum_{i=1}^{m}\left(\frac{\partial u_{i}}{\partial r}+\frac{2}{p-1} \frac{u_{i}}{r}\right)^{2},
$$

where $\frac{\partial}{\partial r}$ is polar derivative.

Note that for the case of single equations, that is when $m=1$, similar monotonicity formulae are given by Pacard in [35] and Weiss in [44, 45]. For the case of systems that is when $m \geq 1$, very recently, Andersson, Shahgholian, Uraltseva and Weiss in [4] provided a monotonicity formula for solutions of system (1.1) when $p=0$ and applied it to study free boundary problems. Let us mention that very similar monotonicity formulae appear in the field of harmonic maps that is

$$
-\Delta u=|\nabla u|^{2} u,
$$

where $u: \mathbb{R}^{n} \rightarrow \mathbb{S}^{m-1}$, see Evans in $[18,19]$ and reference therein.

Case $s=2$. For every $\lambda>0$ and $x_{0} \in \mathbb{R}^{n}$ define

$$
\begin{aligned}
E_{2}\left(u, \lambda, x_{0}\right):= & \lambda^{4 \frac{p+1}{p-1}-n} \int_{B_{\lambda}\left(x_{0}\right)}\left(\frac{1}{2} \sum_{i=1}^{m}\left|\Delta u_{i}\right|^{2}-\frac{1}{p+1}|u|^{p+1}\right) \\
& -\frac{4}{p-1}\left(\frac{p+3}{p-1}-n\right) \lambda^{1+\frac{8}{p-1}-n} \int_{\partial B_{\lambda}\left(x_{0}\right)}|u|^{2} \\
& -\frac{4}{p-1}\left(\frac{p+3}{p-1}-n\right) \frac{d}{d \lambda}\left[\lambda^{\frac{8}{p-1}+2-n} \int_{\partial B_{\lambda}\left(x_{0}\right)}|u|^{2}\right] \\
& +\frac{1}{2} \lambda^{3} \frac{d}{d \lambda}\left[\lambda^{\frac{8}{p-1}+1-n} \int_{\partial B_{\lambda}\left(x_{0}\right)} \sum_{i=1}^{m}\left(\frac{4}{p-1} \lambda^{-1} u_{i}+\frac{\partial u_{i}}{\partial r}\right)^{2}\right] \\
& +\frac{1}{2} \frac{d}{d \lambda}\left[\lambda^{4 \frac{p+1}{p-1}-n} \int_{\partial B_{\lambda}\left(x_{0}\right)} \sum_{i=1}^{m}\left(\left|\nabla u_{i}\right|^{2}-\left|\frac{\partial u_{i}}{\partial r}\right|^{2}\right)\right] \\
& +\frac{1}{2} \lambda^{4 \frac{p+1}{p-1}-n-1} \int_{\partial B_{\lambda}\left(x_{0}\right)} \sum_{i=1}^{m}\left(\left|\nabla u_{i}\right|^{2}-\left|\frac{\partial u_{i}}{\partial r}\right|^{2}\right) .
\end{aligned}
$$

Then the following monotonicity formula holds for the fourth order Lane-Emden system.

Theorem 1.2. Suppose that $n \geq 5, p>\frac{n+4}{n-4}$ and $u$ is a solution of (1.1) when $s=2$. For any $\lambda>0$ and $x_{0} \in \mathbb{R}^{n}$

$$
\frac{d E_{2}\left(u, \lambda, x_{0}\right)}{d \lambda} \geq C \lambda^{\frac{8}{p-1}+2-n} \int_{\partial B_{\lambda}\left(x_{0}\right)} \sum_{i=1}^{m}\left(\frac{4}{p-1} r^{-1} u_{i}+\frac{\partial u_{i}}{\partial r}\right)^{2},
$$

where $E_{2}$ is defined by (1.12) and $C$ is independent from $\lambda$.

Note also that the above monotonicity formula is provided by Davila, Dupaigne, Wang and Wei in [15] for the case of single equations that is when $m=1$. For the case of fractional Laplacian, we provide monotonicity formulae for the extension problems. Assume that $u_{i} \in C^{2 \sigma}\left(\mathbb{R}^{n}\right), \sigma>s>0$ and

$$
\int_{\mathbb{R}^{n}} \frac{\left|u_{i}(y)\right|}{(1+|y|)^{n+2 s}} d y<\infty
$$


for each $1 \leq i \leq m$. The fractional Laplacian of $u_{i}$ when $0<s<1$ denoted by

$$
(-\Delta)^{s} u_{i}(x):=p \cdot v \cdot \int_{\mathbb{R}^{n}} \frac{u_{i}(x)-u_{i}(y)}{|x-y|^{n+2 s}} d y,
$$

is well-defined for every $x \in \mathbb{R}^{n}$. Here $p . v$. stands for the principle value. It is by now standard that the fractional Laplacian can be seen as a Dirichlet-to-Neumann operator for a degenerate but local diffusion operator in the higher-dimensional half-space $\mathbb{R}^{n+1}$, see Caffarelli and Silvestre in [8]. In other words, for $u_{i} \in$ $C^{2 \sigma} \cap L^{1}\left(\mathbb{R}^{n},\left(1+|y|^{n+2 s}\right) d y\right)$ when $\sigma>s$ and $0<s<1$, there exists $v=\left(v_{i}\right)_{i=1}^{m}$ such that $v_{i} \in C^{2}\left(\mathbb{R}_{+}^{n+1}\right) \cap C\left(\overline{\mathbb{R}_{+}^{n+1}}\right)$, $y^{1-2 s} \partial_{y} v_{i} \in C\left(\overline{\mathbb{R}_{+}^{n+1}}\right)$ and

$$
\left\{\begin{aligned}
\nabla \cdot\left(y^{1-2 s} \nabla v_{i}\right) & =0 & & \text { in } \mathbb{R}_{+}^{n+1}, \\
v_{i} & =u_{i} & & \text { on } \partial \mathbb{R}_{+}^{n+1}, \\
-\lim _{y \rightarrow 0} y^{1-2 s} \partial y v_{i} & =\kappa_{s}|v|^{p-1} v_{i} & & \text { on } \partial \mathbb{R}_{+}^{n+1},
\end{aligned}\right.
$$

for the following constant $\kappa_{s}$,

$$
\kappa_{s}:=\frac{\Gamma(1-s)}{2^{2 s-1} \Gamma(s)} .
$$

For the case of $1<s<2$, there are various definitions for the fractional operator $(-\Delta)^{s}$, see $[9,12,24,47]$. From the Fourier transform one can define the fractional Laplacian by

$$
\widehat{(-\Delta)^{s}} u_{i}(\zeta)=|\zeta|^{2 s} \hat{u}_{i}(\zeta)
$$

or inductively by $(-\Delta)^{s}=(-\Delta)^{s-1} o(-\Delta)$. Note that Yang in [47] gave a counterpart of the extension problem by Caffarelli and Silvestre in [8] for the fractional Laplacian $(-\Delta)^{s}$, where $s$ is any positive, noninteger number. In other words, he showed that the higher order fractional Laplacian operator can also be regarded as the Dirichlet-to-Neumann map for an extension function satisfying a higher order elliptic equation in the upper half space with one extra spatial dimension. More precisely, there exists an extension function $v_{i} \in W^{2,2}\left(\mathbb{R}_{+}^{n+1}, y^{b}\right)$ such that

$$
\left\{\begin{aligned}
\Delta_{b}^{2} v_{i} & =0 \text { in } \mathbb{R}_{+}^{n+1} \\
\lim _{y \rightarrow 0} y^{b} \partial_{y} v_{i} & =0 \text { in } \partial \mathbb{R}_{+}^{n+1}, \\
\lim _{y \rightarrow 0} y^{b} \partial_{y} \Delta_{b} v_{i} & =C_{n, s}|v|^{p-1} v_{i} \text { in } \mathbb{R}^{n}
\end{aligned}\right.
$$

where $b:=3-2 s$ and $\Delta_{b} v_{i}:=y^{-b} \operatorname{div}\left(y^{b} \nabla v_{i}\right)$. We refer interested readers to Case and Chang [9] and Chang and Gonzales [12] as well. We are now ready to provide monotonicity formulae for various parameters $s>0$. Case $0<s<1$. Let $v=\left(v_{i}\right)_{i=1}^{m}$ be a solution of the extension problem (1.16). Now define the energy functional for any $\lambda>0$ and $x_{0} \in \partial \mathbb{R}_{+}^{n+1}$ as

$$
\begin{aligned}
E_{s}\left(v, \lambda, x_{0}\right):= & \lambda^{\frac{2 s(p+1)}{p-1}-n}\left(\frac{1}{2} \int_{\mathbb{R}_{+}^{n+1} \cap B_{\lambda}} y^{1-2 s} \sum_{i=1}^{m}\left|\nabla v_{i}\right|^{2} d x d y-\frac{\kappa_{s}}{p+1} \int_{\partial \mathbb{R}_{+}^{n+1} \cap B_{\lambda}}|v|^{p+1} d x\right) \\
& +\lambda^{\frac{2 s(p+1)}{p-1}-n-1} \frac{s}{p+1} \int_{\partial B_{\lambda} \cap \mathbb{R}_{+}^{n+1}} y^{1-2 s}|v|^{2} d \sigma .
\end{aligned}
$$

We are now ready to provide a monotonicity formula for solutions of (1.16) when $0<s<1$.

Theorem 1.3. Suppose that $0<s<1$. Let $v=\left(v_{i}\right)_{i=1}^{m}$ where each $v_{i} \in C^{2}\left(\mathbb{R}_{+}^{n+1}\right) \cap C\left(\overline{\mathbb{R}_{+}^{n+1}}\right)$ be a solution of (1.16) such that $y^{1-2 s} \partial_{y} v_{i} \in C\left(\overline{\mathbb{R}_{+}^{n+1}}\right)$. Then, $E_{s}$ is a nondecreasing function of $\lambda$. Furthermore,

$$
\frac{d E_{s}}{d \lambda}=\lambda^{\frac{2 s(p+1)}{p-1}-n+1} \int_{\partial B\left(x_{0}, \lambda\right) \cap \mathbb{R}_{+}^{n+1}} y^{1-2 s} \sum_{i=1}^{m}\left(\frac{\partial v_{i}}{\partial r}+\frac{2 s}{p-1} \frac{v_{i}}{r}\right)^{2} d \sigma,
$$

where $E_{s}$ provided in (1.20). 
Case $1<s<2$. Suppose that $v=\left(v_{i}\right)_{i=1}^{m}$ is a solution of the extension problem (1.19). Similarly, now define the energy functional for any $\lambda>0$ and $x_{0} \in \partial \mathbb{R}_{+}^{n+1}$ as

$$
\begin{aligned}
E_{s}\left(v, \lambda, x_{0}\right):= & \lambda^{2 s \frac{p+1}{p-1}-n}\left(\int_{\mathbb{R}_{+}^{n+1} \cap B_{\lambda}\left(x_{0}\right)} \frac{1}{2} y^{3-2 s} \sum_{i=1}^{m}\left|\Delta_{b} v_{i}\right|^{2}-\frac{C_{n, s}}{p+1} \int_{\partial \mathbb{R}_{+}^{n+1} \cap B_{\lambda}\left(x_{0}\right)}|v|^{p+1}\right) \\
& -\frac{s}{p-1}\left(\frac{p+2 s-1}{p-1}-n\right) \lambda^{-3+2 s+\frac{4 s}{p-1}-n} \int_{\mathbb{R}_{+}^{n+1} \cap \partial B_{\lambda}\left(x_{0}\right)} y^{3-2 s}|v|^{2} \\
& -\frac{s}{p-1}\left(\frac{p+2 s-1}{p-1}-n\right) \frac{d}{d \lambda}\left[\lambda^{\frac{4 s}{p-1}+2 s-2-n} \int_{\mathbb{R}_{+}^{n+1} \cap \partial B_{\lambda}\left(x_{0}\right)} y^{3-2 s}|v|^{2}\right] \\
& +\frac{1}{2} r^{3} \frac{d}{d \lambda}\left[\lambda_{p^{\frac{4 s}{p-1}+2 s-3-n}} \int_{\mathbb{R}_{+}^{n+1} \cap \partial B_{\lambda}\left(x_{0}\right)} y^{3-2 s} \sum_{i=1}^{m}\left(\frac{2 s}{p-1} \lambda^{-1} v_{i}+\frac{\partial v_{i}}{\partial r}\right)^{2}\right] \\
& +\frac{1}{2} \frac{d}{d \lambda}\left[\lambda^{2 s^{p+1}-n} \int_{\mathbb{R}_{+}^{n+1} \cap \partial B_{\lambda}\left(x_{0}\right)} y^{3-2 s} \sum_{i=1}^{m}\left(\left|\nabla v_{i}\right|^{2}-\left|\frac{\partial v_{i}}{\partial r}\right|^{2}\right)\right] \\
& +\frac{1}{2} \lambda^{2 s \frac{p+1}{p-1}-n-1} \int_{\mathbb{R}_{+}^{n+1} \cap \partial B_{\lambda}\left(x_{0}\right)} y^{3-2 s} \sum_{i=1}^{m}\left(\left|\nabla v_{i}\right|^{2}-\left|\frac{\partial v_{i}}{\partial r}\right|^{2}\right) .
\end{aligned}
$$

Considering the above energy functional, we now provide a monotonicity formula for solutions of (1.19) when $1<s<2$.

Theorem 1.4. Assume that $n>2 s$ and $p>\frac{n+2 s}{n-2 s}$. Let $v=\left(v_{i}\right)_{i=1}^{m}$ be a solution of (1.19) then $E\left(v, \lambda, x_{0}\right)$ is a nondecreasing function of $\lambda>0$. In addition,

$$
\frac{d E_{s}\left(v, \lambda, x_{0}\right)}{d \lambda} \geq C(n, s, p) \lambda^{\frac{4 s}{p-1}+2 s-2-n} \int_{\mathbb{R}_{+}^{n+1} \cap \partial B_{\lambda}\left(x_{0}\right)} y^{3-2 s} \sum_{i=1}^{m}\left(\frac{2 s}{p-1} r^{-1} v_{i}+\frac{\partial v_{i}}{\partial r}\right)^{2},
$$

where $E_{s}$ is given by (1.22) and $C(n, s, p)$ is independent from $\lambda$.

We also refer interested readers to Davila, Dupaigne and Wei in [14] and to Wei and the first author in [24] for a similar monotonicity formula for the case of scalar equations and $0<s<1$ and $1<s<2$, respectively. Before we state our main results let us present some backgrounds regarding classification of solutions of (1.1) in the absence of stability. We provide such classifications for scalar equations and systems separately.

Remark 1.1. Note that monotonicity formulae provided as Theorems 1.1-1.4 hold for the following system with a slightly more general right-hand side,

$$
(-\Delta)^{s} u_{i}=|u|^{p-1}\left(\alpha_{i} u_{i}^{+}+\beta_{i} u_{i}^{-}\right) \quad \text { in } \mathbb{R}^{n},
$$

where $\alpha_{i}$ and $\beta_{i}$ are positive constants for $1 \leq i \leq m$.

Suppose that $m=1$ when (1.1) turns into a single equation. We first consider the local operator cases meaning $s=1$ and $s=2$. Suppose that $s=1$ and the parameter $p$ is in the subcritical case that is when $1<p<p_{S}(n, 1)$ where

$$
p_{S}(n, 1):=\left\{\begin{aligned}
\infty & \text { if } n \leq 2 \\
\frac{n+2}{n-2} & \text { if } n>2
\end{aligned}\right.
$$

For this case, there is a very well-known classification result of Gidas and Spruck in [26] stating that the only nonnegative solution of the Lane-Emden equation (1.1) with $s=1$ is the trivial solution. For the critical case, 
that is when $p=p_{S}(n, 1)$, Caffarelli, Gidas and Spruck in [6] that there is a unique (up to translation and rescaling) positive solution for the Lane-Emden equation. For the fourth order Lane-Emden equation, that is when $s=2$, Wei and $\mathrm{Xu}$ in [43] provided a similar classification result for the subcritical $1<p<p_{S}(n, 2)$ and the critical cases $p=p_{S}(n, 2)$ when

$$
p_{S}(n, 2):=\left\{\begin{aligned}
\infty & \text { if } n \leq 4 \\
\frac{n+4}{n-4} & \text { if } n>4
\end{aligned}\right.
$$

see also [33]. Note that for the fractional Laplacian operator $0<s<1$, such a classification result is given by $\mathrm{Li}[32]$ and Chen-Li-Ou [11] where the critical exponent is

$$
p_{S}(n, s)=\left\{\begin{aligned}
\infty & \text { if } n \leq 2 s, \\
\frac{n+2 s}{n-2 s} & \text { if } n>2 s .
\end{aligned}\right.
$$

For the case of system of equations, that is when $m \geq 2$, Druet, Hebey and Vétois in [17] provided a classification result for solutions of (1.1) where $s=1$ via the moving sphere method. Suppose that $p=\frac{n+2}{n-2}$ and $u=\left(u_{i}\right)_{i=1}^{m}$ is a nonnegative classical solution of (1.1) where $s=1$. Then they proved that there exist $x_{0} \in \mathbb{R}^{n}, \lambda>0, \Lambda \in \mathcal{S}_{+}^{m-1}$ such that

$$
u(x)=\left(\frac{\lambda}{\lambda^{2}+\frac{1}{n(n-2)}\left|x-x_{0}\right|^{2}}\right)^{\frac{n-2}{2}} \Lambda .
$$

We also refer interested readers to $[16,29,30]$ where the authors studied various counterparts of system (1.1). Note that the following singular function

$$
u_{s}(x)=\mathcal{A}|x|^{-\frac{2 s}{p-1}} \text { for } \mathcal{A} \in \mathbb{R}^{m} \text { with }|\mathcal{A}|^{p-1}=2^{2 s} \frac{\Gamma\left(\frac{n}{2}-\frac{s}{p-1}\right) \Gamma\left(s+\frac{s}{p-1}\right)}{\Gamma\left(\frac{s}{p-1}\right) \Gamma\left(\frac{n-2 s}{2}-\frac{s}{p-1}\right)},
$$

solves (1.1) in $\mathbb{R}^{n} \backslash\{0\}$ for a supercritical parameter $p$ that is $p>p_{S}(n, s)$. Before we state our main results, let us define the notion of stable solutions.

Definition 1.1. We say a solution $u$ of (1.1) is stable outside a compact set if there exists $R_{0}>0$ such that

$$
\sum_{i=1}^{m} \int|u|^{p-1} \phi_{i}^{2}+(p-1) \sum_{i, j=1}^{m} \int|u|^{p-3} u_{i} u_{j} \phi_{i} \phi_{j} \leq \sum_{i=1}^{m} \|\left.\phi_{i}\right|_{\dot{H}^{s}\left(\mathbb{R}^{n}\right)} ^{2},
$$

for any $\phi=\left(\phi_{i}\right)_{i=1}^{m}$ where $\phi_{i} \in C_{c}^{\infty}\left(\mathbb{R}^{n} \backslash \overline{B_{R_{0}}}\right)$ for $1 \leq i \leq m$.

Here is our main result.

Theorem 1.5. Suppose $0<s \leq 2$ and $n>2 s$. Let $u$ be a solution of (1.1) that is stable outside a compact set. Then either for $1<p<p_{S}(n, s)$ or for $p>p_{S}(n, s)$ and

$$
p \frac{\Gamma\left(\frac{n}{2}-\frac{s}{p-1}\right) \Gamma\left(s+\frac{s}{p-1}\right)}{\Gamma\left(\frac{s}{p-1}\right) \Gamma\left(\frac{n-2 s}{2}-\frac{s}{p-1}\right)}>\frac{\Gamma\left(\frac{n+2 s}{4}\right)^{2}}{\Gamma\left(\frac{n-2 s}{4}\right)^{2}},
$$

each component $u_{i}$ must be identically zero. For the case of Sobolev critical exponent, that is when $p=p_{S}(n, s)$, a solution $u$ has finite energy that is

$$
\|u\|_{L^{p+1}\left(\mathbb{R}^{n}\right)}^{p+1}=\|u\|_{\dot{H}^{s}\left(\mathbb{R}^{n}\right)}^{2}<\infty .
$$

In this case, if in addition $u$ is stable, then each component $u_{i}$ must be identically zero.

As a direct consequence, the above theorem implies that the only nonnegative solution for system (1.5) when $s=1$ is the trivial solution for dimensions $n<12$ and $n \neq 4$. Here, is how this article is structured. For the rest of this article, we provide a proof for Theorem 1.5 considering various cases for parameter $s>0$. In Section 2, we consider the case $s=1$ that is when the operator in (1.1) is the local Laplacian operator. In Section 3, we let $s=2$ that refers to the bi-Laplacian operator. Lastly, in Section 4, we consider nonlocal cases $0<s<1$ and $1<s<2$. For these non-integer parameters, the operator in (1.1) is a fractional Laplacian operator. For all cases $0<s \leq 2$, we apply blow-down analysis arguments and monotonicity formulae. 


\section{Local Case: Laplacian operator}

In this section we assume that $s=1$. Therefore system (1.1) turns into the following form

$$
-\Delta u_{i}=|u|^{p-1} u_{i} \text { in } \mathbb{R}^{n} .
$$

Note that this is a particular case of system (4.33). For a general nonlinearity $H: \mathbb{R}^{m} \rightarrow \mathbb{R}$ such that $\nabla H \geq 0$, it is proved in [22] that bounded stable solutions of (4.33) are constant when $n \leq 4$. For radial solutions, this is known to hold in more dimensions that is when $n \leq 9$ without any sign assumptions on the nonlinearity, see [21]. In addition it is known that at least for the case of radial solutions the dimension $n=9$ is the optimal dimension. For the rest of this section we prove the following classification of finite Morse index solutions of (2.1).

Theorem 2.1. Suppose that $u=\left(u_{i}\right)_{i=1}^{m}$ is a finite Morse index solution of (2.1) when $m \geq 1$ and $n \geq 3$. Let $1<p<\frac{n+2}{n-2}$ and $\frac{n+2}{n-2}<p<p_{c}(n)$ where

$$
p_{c}(n)=\left\{\begin{aligned}
\infty & \text { if } n \leq 10 \\
\frac{(n-2)^{2}-4 n+8 \sqrt{n-1}}{(n-2)(n-10)} & \text { if } n \geq 11
\end{aligned}\right.
$$

Then each $u_{i}$ must be identically zero. For the Sobolev critical exponent $p=\frac{n+2}{n-2}$, a solution $u$ has finite energy that is

$$
\int_{\mathbb{R}^{n}}|u|^{p+1}=\sum_{i=1}^{m} \int_{\mathbb{R}^{n}}\left|\nabla u_{i}\right|^{2}<\infty .
$$

In this case, if in addition $u$ is stable, then each component $u_{i}$ must be identically zero.

Note that for the case of scalar equations, that is when $m=1$, the above theorem is given by Farina in [20]. He used a Moser iteration type argument for the proof. We refer interested reader to Crandall and Rabinowitz in [13] for a similar approach. To prove the above theorem, we apply a blow-down analysis argument as well as the monotonicity formula given as Theorem 1.1. We now derive a few elliptic estimates.

Lemma 2.1. Suppose that $u$ is a stable solution of (1.1). Then, for any $R>1$

$$
\int_{B_{R}}|u|^{p+1} \leq C R^{n-2 \frac{p+1}{p-1}} \text { and } \sum_{i=1}^{m} \int_{B_{R}}\left|\nabla u_{i}\right|^{2} \leq C R^{n-2 \frac{p+1}{p-1}},
$$

where $C$ is a positive constant that is independent from $R$.

Proof. Test the stability inequality on $\phi_{i}=u_{i} \zeta_{R}$ where $\zeta_{R}: C_{c}^{\infty}\left(\mathbb{R}^{n}\right) \rightarrow \mathbb{R}$ and $\zeta_{R} \equiv 1$ on $B_{R}$ and $\zeta_{R} \equiv 0$ on $\mathbb{R}^{n} \backslash B_{2 R}$ with $\left\|\zeta_{R}\right\|_{L^{\infty}\left(B_{2 R} \mid B_{R}\right)} \leq C R^{-1}$. Now multiply both sides of (1.1) with $u_{i} \zeta_{R}^{2}$ and integrate by parts. Equating the inequalities that one gets from this and from stability finishes the proof.

Applying the Hölder's inequality, we get the following $L^{2}$ estimate.

Corollary 2.1. Suppose that $u$ is a stable solution of (1.1). Then, for any $R>1$

$$
\int_{B_{R}}|u|^{2} \leq C R^{n-\frac{4}{p-1}},
$$

where $C$ is a positive constant that is independent from $R$.

In this part, we present a classification of stable homogeneous solutions. This is a key point in our proof of Theorem 2.1. 
Theorem 2.2. Suppose that $u=\left(u_{i}\right)_{i=1}^{m}$ for $u_{i}=r^{-\frac{2}{p-1}} \psi_{i}(\theta)$ is a stable solution of (1.1). Then, each $\psi_{i}$ is identically zero provided $\frac{n+2}{n-2}<p<p_{c}(n)$ where $p_{c}(n)$ is given by (2.2).

Proof. We omit the proof here, since a similar argument will be provided in the proof of Theorem 3.2 for the fourth order case.

Proof of Theorem 2.1. The proof is based on a blow-down analysis and it relies on the monotonicity formula provided as Theorem 1.1. We omit the details, since similar arguments will be provided for the poof of Theorem 3.1. For the case of Sobolev critical exponent, one can conclude the result via applying the Pohozaev indentity.

\section{Local Case: Bi-Laplacian Operator}

In this section, we consider the following fourth order system

$$
\Delta^{2} u_{i}=|u|^{p-1} u_{i} \text { in } \mathbb{R}^{n}
$$

This section is devoted to the proof of the following theorem.

Theorem 3.1. Suppose that $u=\left(u_{i}\right)_{i=1}^{m}$ is a finite Morse index solution of (3.1) when $m \geq 1$ and $n \geq 5$. Let $1<p<\frac{n+4}{n-4}$ and $\frac{n+4}{n-4}<p<\bar{p}_{c}(n)$ where

$$
\bar{p}_{c}(n)=\left\{\begin{aligned}
\infty & \text { if } n \leq 12, \\
\frac{n+2-\sqrt{n^{2}+4-n \sqrt{n^{2}-8 n+32}}}{n-6-\sqrt{n^{2}+4-n \sqrt{n^{2}-8 n+32}}} & \text { if } n \geq 13 .
\end{aligned}\right.
$$

Then, each $u_{i}$ must be identically zero. For the Sobolev critical exponent $p=\frac{n+4}{n-4}$, a solution $u$ has finite energy that is

$$
\int_{\mathbb{R}^{n}}|u|^{p+1}=\sum_{i=1}^{m} \int_{\mathbb{R}^{n}}\left|\Delta u_{i}\right|^{2}<\infty .
$$

In this case, if in addition $u$ is stable, then each component $u_{i}$ must be identically zero.

In order to prove the above theorem, we are required to establish some a priori estimates on solutions.

Lemma 3.1. Suppose that $u=\left(u_{i}\right)_{i=1}^{m}$ be a smooth stable solution of (3.1) and set $w=\left(w_{i}\right)_{i=1}^{m}$ where $w_{i}=\Delta u_{i}$. Then the following estimate holds,

$$
\int_{\mathbb{R}^{n}}\left(|w|^{2}+|u|^{p+1}\right) \zeta^{2} \leq C \int_{\mathbb{R}^{n}}|u|^{2}\left(|\nabla \Delta \zeta||\nabla \zeta|+|\Delta \zeta|^{2}+\left.|\Delta| \nabla \zeta\right|^{2} \mid\right)+|u||w||\nabla \zeta|^{2},
$$

for a test function $\zeta: C_{c}^{\infty}\left(\mathbb{R}^{n}\right) \rightarrow \mathbb{R}$.

Proof. Test the stability inequality on $u_{i} \zeta$ where $\zeta$ is a test function then multiply both sides of the $i^{\text {th }}$ equation of (1.1) with $u_{i} \zeta^{2}$. Equating these completes the proof.

Applying an appropriate test function yields the following estimate.

Corollary 3.1. Under the same assumptions as in (3.1), there exists a constant $C$ such that

$$
\int_{B_{R}(x)}|w|^{2}+|u|^{p+1} \leq C R^{-4} \int_{B_{2 R}(x) \backslash B_{R}(x)}|u|^{2}+C R^{-2} \int_{B_{2 R}(x) \backslash B_{R}(x)}|u||w|,
$$


and therefore

$$
\int_{B_{R}(x)}|w|^{2}+|u|^{p+1} \leq C R^{n-4 \frac{p+1}{p-1}}
$$

Proof. Set the test function $\zeta_{R} \in C_{c}^{1}\left(\mathbb{R}^{n}\right)$ in (3.1) where $0 \leq \zeta_{R} \leq 1$ being the following test function

$$
\zeta_{R}(x)= \begin{cases}1, & \text { if }|x|<R, \\ 0, & \text { if }|x|>2 R\end{cases}
$$

satisfying $\left\|\nabla \zeta_{R}\right\|_{\infty} \leq R^{-1}$ and $\left\|\Delta \zeta_{R}\right\|_{\infty} \leq R^{-2}$

We now provide classification of stable homogeneous solutions. Note first that the following Hardy-Rellich inequality with the best constant holds. Suppose that $h: C_{c}^{2}\left(\mathbb{R}^{n}\right) \rightarrow \mathbb{R}$ then

$$
\int_{\mathbb{R}^{n}}|\Delta h|^{2} d x \geq \frac{n^{2}(n-4)^{2}}{16} \int_{\mathbb{R}^{n}} \frac{h^{2}}{|x|^{4}} d x .
$$

This inequality implies that the singular solution given by (1.29) is stable if and only if

$$
p|\mathcal{A}|^{p-1}=p \alpha(\alpha+2)(n-\alpha-2)(n-\alpha-4) \leq \frac{n^{2}(n-4)^{2}}{16},
$$

where $\alpha:=\frac{4}{p-1}$ and $\mathcal{A}$ given in (1.29).

Theorem 3.2. Suppose that $u=\left(u_{i}\right)_{i=1}^{m}$ for $u_{i}=r^{-\frac{4}{p-1}} \psi_{i}(\theta)$ is a stable solution of (3.1). Then, each $\psi_{i} \equiv 0$ provided $\frac{n+4}{n-4}<p<\bar{p}_{c}(n)$ where $\bar{p}_{c}(n)$ given by (3.2).

Proof. It is straightforward to see that $\psi=\left(\psi_{i}\right)_{i=1}^{m}$ satisfies

$$
\Delta_{\theta}^{2} \psi_{i}-\alpha \Delta_{\theta} \psi_{i}+\beta \psi_{i}=|\psi|^{p-1} \psi_{i}
$$

for

$$
\alpha:=(q+2)(n-4-q)+q(n-2-q) \text { and } \beta:=q(q+2)(n-4-q)(n-2-q),
$$

where $q:=\frac{4}{p-1}$. Multiplying both sides of (3.9) with $\psi_{i}$ and integrating over $\mathcal{S}^{n-1}$, we conclude

$$
\sum_{i=1}^{m} \int_{\mathcal{S}^{n-1}}\left[\left|\Delta \psi_{i}\right|^{2}+\alpha\left|\nabla \psi_{i}\right|^{2}\right]+\beta \int_{\mathcal{S}^{n-1}}|\psi|^{2}=\int_{\mathcal{S}^{n-1}}|\psi|^{p+1} .
$$

We now test the stability inequality (1.30) on $\phi=\left(\phi_{i}\right)_{i=1}^{m}$ for $\phi_{i}:=r^{-\frac{n-4}{2}} \psi_{i}(\theta) \eta_{\epsilon}(r)$. Here, $\eta_{\epsilon}$ is a standard cut-off function $\eta_{\epsilon} \in C_{c}^{1}\left(\mathbb{R}_{+}\right)$at the origin and at infinity that is $\eta_{\epsilon}=1$ for $\epsilon<r<\epsilon^{-1}$ and $\eta_{\epsilon}=0$ for either $r<\epsilon / 2$ or $r>2 / \epsilon$. Applying similar ideas provided in [24], we get

$$
p \int_{\mathcal{S}^{n-1}}|\psi|^{p+1} \leq \sum_{i=1}^{m} \int_{\mathcal{S}^{n-1}}\left[\left|\Delta \psi_{i}\right|^{2}+\frac{n(n-4)}{2}\left|\nabla \psi_{i}\right|^{2}\right]+\frac{n^{2}(n-4)^{2}}{16} \int_{\mathcal{S}^{n-1}}|\psi|^{2} .
$$

Combining (3.11) and (3.12) we get

$$
(p-1) \sum_{i=1}^{m} \int_{\mathcal{S}^{n-1}}\left[\left|\Delta \psi_{i}\right|^{2}+\left(p \alpha-\frac{n(n-4)}{2}\right)\left|\nabla \psi_{i}\right|^{2}\right]+\left(p \beta-\frac{n^{2}(n-4)^{2}}{16}\right)|\psi|^{2} \leq 0 .
$$

Note the coefficients $p-1, p \alpha-\frac{n(n-4)}{2}$ and $p \beta-\frac{n^{2}(n-4)^{2}}{16}$ are positive when $\frac{n+4}{n-4}<p<p_{c}(n)$ where $p_{c}(n)$ is given by (3.2). 
Proof of Theorem 3.1. The proof of the Sobolev critical case relies on applying the Pohozaev identity and we omit it here. We now provide a sketch of the proof when $p>\frac{n+4}{n-4}$ in a few steps.

Step $1 . \lim _{r \rightarrow \infty} E(u, r, 0)<\infty$. Note that the energy $E(u, r, 0)$ is nondecreasing in $r$, as given in Theorem 4.14. Therefore,

$$
E(u, r, 0) \leq r^{-1} \int_{r}^{2 r} E(u, t, 0) d t \leq r^{-2} \int_{r}^{2 r} \int_{t}^{t+r} E(u, \lambda, 0) d \lambda d t .
$$

Applying estimates given in Corollary 3.1 imply that the right-hand side of (3.14) is bounded.

Step 2. Define $u_{i}^{\lambda}(x)=\lambda^{\frac{4}{p-1}} u_{i}(\lambda x)$ for each $1 \leq i \leq m$ where $u=\left(u_{i}\right)_{i=1}^{m}$ is a stable solution of (1.1). Then $u_{i}^{\lambda} \rightarrow u_{i}^{\infty}$ where $u_{i}^{\infty} \in W_{\text {loc }}^{2,2}\left(\mathbb{R}^{n}\right) \cap L_{\text {loc }}^{p+1}\left(\mathbb{R}^{n}\right)$ and $u^{\infty}=\left(u_{i}^{\infty}\right)_{i=1}^{m}$ is a stable solution of (1.1). Set $w_{i}^{\lambda}(x):=\lambda^{\frac{4}{p-1}+2} w_{i}(\lambda x)$. From Corollary 3.1, we have

$$
\int_{B_{r}(x)}\left|w^{\lambda}\right|^{2}+\left|u^{\lambda}\right|^{p+1} \leq C r^{n-4 \frac{p+1}{p-1}} .
$$

From elliptic estimates, up to a subsequence, $u_{i}^{\lambda} \rightarrow u_{i}^{\infty}$ for each $1 \leq i \leq m$ weakly in $W_{l o c}^{2,2}\left(\mathbb{R}^{n}\right) \cap L_{l o c}^{p+1}\left(\mathbb{R}^{n}\right)$. From compactness embeddings and applying interpolation we arrive at $u_{i}^{\lambda} \rightarrow u_{i}^{\infty}$ in $L_{l o c}^{q}\left(\mathbb{R}^{n}\right)$ for any $q \in[1, p+1)$. Note also that $u^{\infty}$ is a stable solution, since $u^{\lambda}$ is a stable solution and we can send $\lambda$ to infinity.

Step 3. $u^{\infty}$ is a homogeneous solution. This is a direct consequence of the monotonicity formula and the following fact

$$
\lim _{\lambda \rightarrow \infty}\left[E\left(u^{\lambda}, R, 0\right)-E\left(u^{\lambda}, r, 0\right)\right]=0 .
$$

The left-hand side of (3.16) is bounded from below by

$$
\begin{aligned}
E\left(u^{\lambda}, R, 0\right)-E\left(u^{\lambda}, r, 0\right) & \geq C(n, p) \sum_{i=1}^{m} \int_{B_{R} \backslash B_{r}}\left(\frac{4}{p-1}|x|^{-1} u_{i}^{\lambda}+\frac{\partial u_{i}^{\lambda}}{\partial r}\right)^{2}|x|^{\frac{8}{p-1}+2-n} d x \\
& =C(n, p) \sum_{i=1}^{m} \int_{B_{R} \backslash B_{r}}\left(\frac{4}{p-1}|x|^{-1} u_{i}^{\infty}+\frac{\partial u_{i}^{\infty}}{\partial r}\right)^{2}|x|^{\frac{8}{p-1}+2-n} d x .
\end{aligned}
$$

This implies that for each $1 \leq i \leq m$, we have

$$
u_{i}^{\infty}(x)=|x|^{-\frac{4}{p-1}} u_{i}^{\infty}\left(\frac{x}{|x|}\right) .
$$

This completes the proof of this step.

Step 4. $\lim _{r \rightarrow \infty} E(u, r, 0)=0$. Since each $u_{i}^{\infty}$ is a homogenous function, Theorem 3.2 implies that $u^{\infty}=0$. Therefore, $\lim _{\lambda \rightarrow \infty} u_{i}^{\lambda}=0$ strongly in $L^{2}\left(B_{4}\right)$ for each $i=1, \cdots, m$ that is

$$
\lim _{\lambda \rightarrow \infty} \int_{B_{4}}\left|u_{i}^{\lambda}\right|^{2}=0 \text { and } \lim _{\lambda \rightarrow \infty} \int_{B_{4}}\left|u_{i}^{\lambda} w_{i}^{\lambda}\right|=0 .
$$

Applying Corollary 3.1, we conclude

$$
\lim _{\lambda \rightarrow \infty} \int_{B_{4}}\left|w^{\lambda}\right|^{2}+\left|u^{\lambda}\right|^{p+1}=0 .
$$

On the other hand, there exists $r_{0}>0$ such that

$$
\lim _{i \rightarrow \infty}\left\|u^{\lambda_{i}}\right\|_{W^{2,2}\left(\partial B_{r_{0}}\right)}=0 .
$$

This implies that

$$
\lim _{i \rightarrow \infty} E\left(u, \lambda_{i} r_{0}, 0\right)=\lim _{i \rightarrow \infty} E\left(u^{\lambda_{i}}, r_{0}, 0\right)
$$

The fact that $E$ is nondecreasing, that is given as a monotonicity formula in Theorem 1.2, completes the proof. 


\section{Nonlocal Case: Lower and higher order fractional Laplacian}

In this section, we consider system (1.1) with the fractional Laplacian operator $(-\Delta)^{s}$ where $s \in(0,2)$ for $s \neq 1$ and we establish Theorem 1.5. We first note that the following Hardy inequality holds for $n>2 s$

$$
\int_{\mathbb{R}^{n}}|\xi|^{2 s}|\hat{h}|^{2} d \xi>\Lambda_{n, s} \int_{\mathbb{R}^{n}}|x|^{-2 s} h^{2} d x
$$

for any $h \in C_{c}^{\infty}\left(\mathbb{R}^{n}\right)$ where the optimal constant is given by

$$
\Lambda_{n, s}=2^{2 s} \frac{\Gamma\left(\frac{n+2 s}{4}\right)^{2}}{\Gamma\left(\frac{n-2 s}{4}\right)^{2}} .
$$

Note that $\Lambda_{n, 2}=\frac{n^{2}(n-4)^{2}}{16}$ meaning (4.1) recovers (3.7) for $s=2$. For more information interested readers are encouraged to see [29] by Herbst (and also [46]). We now provide a classification result for homogeneous solutions. Note that for the case of scalar equations, that is when $m=1$, this classification was given in [24]. Note also that the proof is valid regardless of magnitude of the parameter $s$.

Theorem 4.1. Suppose $u_{i}=r^{-\frac{2 s}{p-1}} \psi_{i}(\theta)$ is a stable solution of (1.1) for $s \in(0,2)$ and $s \neq 1$. Then, each $\psi_{i}$ vanishes identically, provided $p>\frac{n+2 s}{n-2 s}$ and

$$
p \frac{\Gamma\left(\frac{n}{2}-\frac{s}{p-1}\right) \Gamma\left(s+\frac{s}{p-1}\right)}{\Gamma\left(\frac{s}{p-1}\right) \Gamma\left(\frac{n-2 s}{2}-\frac{s}{p-1}\right)}>\frac{\Gamma\left(\frac{n+2 s}{4}\right)^{2}}{\Gamma\left(\frac{n-2 s}{4}\right)^{2}} .
$$

Proof. Since $u=\left(u_{i}\right)_{i=1}^{m}$ is a solution of (1.1), each $\psi_{i}$ satisfies

$$
\begin{aligned}
|x|^{-\frac{2 p s}{p-1}}|\psi|^{p-1}(\theta) \psi_{i}(\theta)= & \int \frac{|x|^{-\frac{2 s}{p-1}} \psi_{i}(\theta)-|y|^{-\frac{2 s}{p-1}} \psi_{i}(\sigma)}{|x-y|^{n+2 s}} d y \\
= & |x|^{-\frac{2 p s}{p-1}}\left[\int \frac{\psi_{i}(\theta)-t^{-\frac{2 s}{p-1}} \psi_{i}(\theta)}{\left(t^{2}+1-2 t<\theta, \sigma>\right)^{\frac{n+2 s}{2}}} t^{n-1} d t d \sigma\right. \\
& \left.+\int \frac{t^{-\frac{2 s}{p-1}}\left(\psi_{i}(\theta)-\psi_{i}(\sigma)\right.}{\left(t^{2}+1-2 t<\theta, \sigma>\right)^{\frac{n+2 s}{2}}} t^{n-1} d t d \sigma\right],
\end{aligned}
$$

where we have used the change of variable $|y|=t|x|$. Simplifying the above, for each $i$, we obtain

$$
\psi_{i}(\theta) A_{n, s}+\int_{\mathbb{S}^{n-1}} K_{\frac{2 s}{p-1}}(\langle\theta, \sigma\rangle)\left(\psi_{i}(\theta)-\psi_{i}(\sigma)\right) d \sigma=|\psi|^{p-1}(\theta) \psi_{i}(\theta),
$$

where

$$
A_{n, s}:=\int_{0}^{\infty} \int_{\mathbb{S}^{n-1}} \frac{1-t^{-\frac{2 s}{p-1}}}{\left(t^{2}+1-2 t<\theta, \sigma>\right)^{\frac{n+2 s}{2}}} t^{n-1} d \sigma d t,
$$

and

$$
K_{\frac{2 s}{p-1}}(<\theta, \sigma>):=\int_{0}^{\infty} \frac{t^{n-1-\frac{2 s}{p-1}}}{\left(t^{2}+1-2 t<\theta, \sigma>\right)^{\frac{n+2 s}{2}}} d t .
$$

Multiplying (4.5) with $\psi_{i}$ and integrating we get

$$
\int_{\mathbb{S}^{n-1}}|\psi|^{2}(\theta) A_{n, s}+\int_{\mathbb{S}^{n-1}} K_{\frac{2 s}{p-1}}(\langle\theta, \sigma\rangle)|\psi(\theta)-\psi(\sigma)|^{2} d \theta d \sigma=\int_{\mathbb{S}^{n-1}}|\psi|^{p+1}(\theta) d \theta .
$$

We now test the stability inequality (1.30) for $\phi_{i}(x)=r^{-\frac{n-2 s}{2}} \psi_{i}(\theta) \eta_{\epsilon}(r)$ and $u_{i}=r^{-\frac{2 s}{p-1}} \psi_{i}(\theta)$ with the same $\eta_{\epsilon}(r)$ as the one given in the proof of Theorem 3.2. Applying similar ideas provided in [24], we conclude

$$
\Lambda_{n, s} \int_{\mathbb{S}^{n-1}}|\psi|^{2}+\int_{\mathbb{S}^{n-1}} K_{\frac{n-2 s}{2}}(\langle\theta, \sigma\rangle)|\psi(\theta)-\psi(\sigma)|^{2} d \sigma \geq p \int_{\mathbb{S}^{n-1}}|\psi|^{p+1},
$$


when

$$
\Lambda_{n, s}:=\int_{0}^{\infty} \int_{\mathbb{S}^{n-1}} \frac{1-t^{\frac{n-2 s}{2}}}{\left(t^{2}+1-2 t<\theta, \sigma>\right)^{\frac{n+2 s}{2}}} t^{n-1} d \sigma d t .
$$

Combining (4.9) and (4.8), we end up with

$$
\left(\Lambda_{n, s}-p A_{n, s}\right) \int_{\mathbb{S}^{n-1}}|\psi|^{2}+\int_{\mathbb{S}^{n-1}}\left(K_{\frac{n-2 s}{2}}-p K_{\frac{2 s}{p-1}}\right)(\langle\theta, \sigma\rangle)|\psi(\theta)-\psi(\sigma)|^{2} d \sigma \geq 0 .
$$

The fact that $K_{\alpha}$ is decreasing in $\alpha$ implies $K_{\frac{n-2 s}{2}}<K_{\frac{2 s}{p-1}}$ for $p>\frac{n+2 s}{n-2 s}$. Therefore, $K_{\frac{n-2 s}{2}}-p K_{\frac{2 s}{p-1}}<0$. On the other hand the assumption of the theorem implies that $\Lambda_{n, s}^{p-1}-p A_{n, s}<0$. Therefore, each $\psi_{i}$ vanishes identically. This completes the proof.

\subsection{Lower Order Fractional Laplacian Operator}

In this part, we show that Theorem 1.5 holds when $0<s<1$. To do so we provide the following estimate first.

Lemma 4.1. Suppose that $p \neq \frac{n+2 s}{n-2 s}$. Let $u$ be a solution of (1.1) that is stable outside a ball $B_{R_{0}}$ and $v$ satisfies (1.16). Then there exists a constant $C>0$ such that

$$
\int_{B_{R}} y^{1-2 s}|v|^{2} \leq C R^{n+2-2 s \frac{p+1}{p-1}}
$$

for any $R>3 R_{0}$.

Lemma 4.2. Let $u$ be a solution of (1.1) that is stable outside a ball $B_{R_{0}}$ and $v$ satisfies (1.16). Then there exists a positive constant $C$ such that

$$
\int_{B_{R} \cap \partial \mathbb{R}_{+}^{n+1}}|v|^{p+1}+\sum_{i=1}^{m} \int_{B_{R} \cap \mathbb{R}_{+}^{n+1}} y^{1-2 s}\left|\nabla v_{i}\right|^{2} \leq C R^{n-2 s \frac{p+1}{p-1}} .
$$

Proof of Theorem 1.5 when $0<s<1$. We omit the proof here since arguments are very similar to the ones which will be provided for the case of $1<s<2$.

\subsection{Higher Order Fractional Laplacian Operator}

As the last past of this section, we shall restrict ourselves to the case $1<s<2$. Let us start with the following integral estimate on stable solutions.

Lemma 4.3. Let $u$ be a solution of (1.1) that is stable outside a ball $B_{R_{0}}$ and $v$ satisfies (1.19). Then there exists a positive constant $C$ such that

$$
\begin{aligned}
\int_{\partial \mathbb{R}_{+}^{n+1}}|v|^{p+1} \eta^{2}+\sum_{i=1}^{m} \int_{\mathbb{R}_{+}^{n+1}} y^{b}\left|\Delta_{b} v_{i}\right|^{2} \eta^{2} \leq & C \int_{\mathbb{R}_{+}^{n+1}} y^{b}|v|^{2}\left(\left|\Delta_{b} \eta\right|^{2}+\left.\left|\Delta_{b}\right| \nabla \eta\right|^{2}|+| \nabla \eta \cdot \nabla \Delta_{b} \eta \mid\right) \\
& +C \sum_{i=1}^{m} \int_{\mathbb{R}_{+}^{n+1}} y^{b}\left|v_{i}\right|\left|\Delta_{b} v_{i}\right||\nabla \eta|^{2}
\end{aligned}
$$


Proof. Here is a sketch of the proof. Multiply the $i^{t h}$ equation of (1.19) with $y^{b} u_{i} \eta^{2}$ where $\eta$ is a test function to get

$$
C_{n, s} \int_{\partial \mathbb{R}_{+}^{n+1}}|v|^{p+1} \eta^{2}=\sum_{i=1}^{m} \int_{\mathbb{R}_{+}^{n+1}} y^{b} \Delta_{b} v_{i} \Delta_{b}\left(v_{i} \eta^{2}\right) .
$$

Note also that for each $i$ these identities hold for a test function $\eta$

$$
\begin{aligned}
\Delta_{b} v_{i} \Delta_{b}\left(v_{i} \eta^{2}\right)-\left|\Delta_{b}\left(v_{i} \eta\right)\right|^{2} & =-v_{i}^{2}\left|\Delta_{b} \eta\right|^{2}+2 v_{i} \Delta_{b} v_{i}|\nabla \eta|^{2}-4\left|\nabla v_{i} \cdot \nabla \eta\right|^{2}-4 v_{i} \Delta_{b} \eta \nabla v_{i} \cdot \nabla \eta, \\
\Delta_{b}\left(v_{i} \eta\right) & =\eta \Delta_{b} v_{i}+v_{i} \Delta_{b} \eta+2 \nabla v_{i} \cdot \nabla \eta .
\end{aligned}
$$

Applying these identifies together with (4.16) one can see that

$$
\begin{aligned}
C_{n, s} \int_{\partial \mathbb{R}_{+}^{n+1}}|v|^{p+1} \eta^{2}= & \sum_{i=1}^{m} \int_{\mathbb{R}_{+}^{n+1}} y^{b}\left|\Delta_{b}\left(v_{i} \eta\right)\right|^{2}+2 \sum_{i=1}^{m} \int_{\mathbb{R}_{+}^{n+1}} y^{b} v_{i} \Delta_{b} v_{i}|\nabla \eta|^{2} \\
& -4 \sum_{i=1}^{m} \int_{\mathbb{R}_{+}^{n+1}} y^{b}\left|\nabla v_{i} \cdot \nabla \eta\right|^{2}+\int_{\mathbb{R}_{+}^{n+1}} y^{b}|v|^{2}\left(\left|\Delta_{b} \eta\right|^{2}+2 \nabla \eta \cdot \nabla \Delta_{b} \eta\right) .
\end{aligned}
$$

Testing the stability inequality (1.30) on $\phi_{i}=u_{i} \eta$ and applying (4.19) one can finish the proof.

One can set $\eta$ to be the standard test function to get the following estimate.

Corollary 4.1. With the same assumption as Lemma 4.3. Then there exists a positive constant $C$ such that

$$
\int_{B_{R} \cap \partial \mathbb{R}_{+}^{n+1}}|v|^{p+1}+\sum_{i=1}^{m} \int_{B_{R} \cap \mathbb{R}_{+}^{n+1}} y^{b}\left|\Delta_{b} v_{i}\right|^{2} \leq C R^{-4} \int_{B_{R} \cap \mathbb{R}_{+}^{n+1}} y^{b}|v|^{2} .
$$

Here we provide more decay estimates of solutions. These lemmata are main tools in our proof of Theorem 1.5. We omit the proofs here and we refer interested readers to see the proof of Lemma 4.5-4.6 in [24] where similar arguments are applied.

Lemma 4.4. Suppose that $p \neq \frac{n+2 s}{n-2 s}$. Let $u$ be a solution of (1.1) that is stable outside a ball $B_{R_{0}}$ and $v$ satisfies (1.19). Then there exists a constant $C>0$ such that

$$
\int_{B_{R}} y^{b}|v|^{2} \leq C R^{n+4-2 s \frac{p+1}{p-1}},
$$

for any $R>3 R_{0}$.

Lemma 4.5. Let $u$ be a solution of (1.1) that is stable outside a ball $B_{R_{0}}$ and $v$ satisfies (1.19). Then there exists a positive constant $C$ such that

$$
\int_{B_{R} \cap \partial \mathbb{R}_{+}^{n+1}}|v|^{p+1}+\sum_{i=1}^{m} \int_{B_{R} \cap \mathbb{R}_{+}^{n+1}} y^{b}\left|\Delta_{b} v_{i}\right|^{2} \leq C R^{n-2 s \frac{p+1}{p-1}} .
$$

Proof of Theorem 1.5 when $1<s<2$. The proof of the case $p=p_{S}(n, s)$ is based on the Pohozaev identity provided in $[24,38]$, and we omit it here. The proof is based on the monotonicity formula that is Theorem 1.4 and a blow-down analysis.

Step 1. The energy is bounded, that is $\lim _{\lambda \rightarrow \infty} E(v, 0, \lambda)<\infty$. This is a direct consequence of the monotonicity formula and Lemma 4.4 and Lemma 4.5. Similar to the proof of Theorem 3.1 we have

$$
E(v, \lambda, 0) \leq \lambda^{-2} \int_{\lambda}^{2 \lambda} \int_{t}^{t+\lambda} E(v, y, 0) d y d t .
$$


Lemma 4.5 and Lemma 4.4 imply that the right-hand side of (4.23) is bounded.

Step 2. The sequence $v_{i}^{\lambda}$ converges weakly in $H_{l o c}^{1}\left(\mathbb{R}^{n}, y^{3-2 s} d x d y\right)$ to a function $v_{i}^{\infty}$ where each $v_{i}^{\infty}$ is homogeneous for $1 \leq i \leq m$ and therefore they are zero. Note that the convergence part is a direct consequence of the elliptic estimates. We now show that each $v_{i}^{\infty}$ is homogeneous. From the boundedness of the energy we have

$$
\lim _{k \rightarrow \infty}\left[E\left(v, R_{2} \lambda_{k}, 0\right)-E\left(v, R_{1} \lambda_{k}, 0\right)\right]=0 .
$$

From this and applying the scaling invariance of the energy and also the monotonicity formula we get

$$
\begin{aligned}
& 0=\liminf _{k \rightarrow \infty} \sum_{i=1}^{m} \int_{\left(B_{R_{2}} \backslash B_{R_{1}}\right) \cap \mathbb{R}_{+}^{n+1}} y^{3-2 s} r^{\frac{4 s}{p-1}+2 s-2-n}\left(\frac{2 s}{p-1} r^{-1} v_{i}^{\lambda_{k}}+\frac{\partial v_{i}^{\lambda_{k}}}{\partial r}\right)^{2} d y d x \\
& \geq \sum_{i=1}^{m} \int_{\left(B_{R_{2}} \backslash B_{R_{1}}\right) \cap \mathbb{R}_{+}^{n+1}} y^{3-2 s} r^{\frac{4 s}{p-1}+2 s-2-n}\left(\frac{2 s}{p-1} r^{-1} v_{i}^{\infty}+\frac{\partial v_{i}^{\infty}}{\partial r}\right)^{2} d y d x,
\end{aligned}
$$

since we have the weak convergence of $\left(v_{i}^{\lambda_{k}}\right)$ to $v_{i}^{\infty}$ in $H_{l o c}^{1}\left(\mathbb{R}^{n}, y^{3-2 s} d y d x\right)$. Therefore,

$$
\frac{2 s}{p-1} r^{-1} v_{i}^{\infty}+\frac{\partial v_{i}^{\infty}}{\partial r}=0 \text { a.e. in } \mathbb{R}_{+}^{n+1},
$$

for each $1 \leq i \leq m$.

Step 3. $\lim _{\lambda \rightarrow \infty} E(v, \lambda, 0)=0$. Note that the monotonicity formula implies that

$$
E(v, \lambda, 0) \leq \lambda^{-1} \int_{\lambda}^{2 \lambda} E(t) d t \leq \sup _{[\lambda, 2 \lambda]} I+C \lambda^{-n-1+\frac{2 s(p+1)}{p-1}} \int_{B_{2 \lambda} \backslash B_{\lambda}}|v|^{2},
$$

where

$$
I(v, \lambda)=I\left(v^{\lambda}, 1\right)=\frac{1}{2} \sum_{i=1}^{m} \int_{\mathbb{R}_{+}^{n+1} \cap B_{1}} y^{3-2 s}\left|\Delta_{b} v_{i}^{\lambda}\right|^{2} d x d y-\frac{\kappa_{s}}{p+1} \int_{\partial \mathbb{R}_{+}^{n+1} \cap B_{1}}\left|v^{\lambda}\right|^{p+1} d x .
$$

Note that $\lim \lambda \rightarrow \infty I(v, \lambda)=0$. On the other hand, from the fact that $u^{\infty}$ is a homogenous solution we have

$$
\lim _{\lambda \rightarrow \infty} u_{i}^{\lambda}=0 \text {, }
$$

strongly in $L^{2}\left(B_{4}\right)$. Therefore,

$$
\lim _{\lambda \rightarrow \infty} \int_{B_{4}}\left|u^{\lambda}\right|^{2}=0 .
$$

This implies that $\lim _{\lambda \rightarrow \infty} E(v, \lambda, 0)=0$ and completes the proof.

We end this section with the following open problem in regards to monotonicity formulae for the LaneEden system.

Open Problem 1. Consider the Lane-Emden system for any parameters $s>0$ and $1<q<p$

$$
\left\{\begin{array}{l}
(-\Delta)^{s} u=v^{p} \text { in } \mathbb{R}^{n}, \\
(-\Delta)^{s} v=u^{q} \text { in } \mathbb{R}^{n} .
\end{array}\right.
$$

Proving a monotonicity formula for the above system, similar to the ones given in Theorem 1.1-1.4, seems more challenging to derive. Note that for the case of $1<q=p$ and $1=q<p$ such monotonicity formulae are known, see the introduction. Needless to mention that the Lane-Emden system (4.32) is not a gradient system, meaning that it is not of the following form

$$
(-\Delta)^{s} u=\nabla H(u) \text { in } \mathbb{R}^{n}
$$

where $u: \mathbb{R}^{n} \rightarrow \mathbb{R}^{m}$.

Lastly, let us mention that in [23] we apply monotonicity formulae derived in this article to prove regularity of free boundaries and partial regularity of weak solutions for certain coupled elliptic systems. 
Acknowledgement: The first author appreciates H. Shahgholian and J. Wei for their hospitality during his visits to KTH and UBC, and he is thankful to E. Hebey for his talk at the conference on the occasion of Michael Struwe's 60th birthday at ETH.

The first author is partially supported by University of Texas at San Antonio Start-up Grant. The second author was partially supported by Swedish Research Council.

\section{References}

[1] H.W. Alt, L.A. Caffarelli and A. Friedman, Variational problems with two phases and their free bound-aries, Trans. Amer. Math. Soc., 282(2) (1984) pp. 431- 461.

[2] W. K. Allard. On the first variation of a varifold. Ann. of Math., 95 (1972) pp. 417-491.

[3] J. Andersson, Optimal regularity for the Signorini problem and its free boundary, Inventiones Math., 204 (2016) pp. 1-82.

[4] J. Andersson, H. Shahgholian, N. N. Uraltseva. and G. S. Weiss, Equilibrium points of a singular cooperative system with free boundary, Advances in Mathematics, 280 (2015) pp. 743-771.

[5] B. Ou, Global solutions to a free boundary problem. Comm. Partial Differential Equations, 19 (1994) pp. 369-397.

[6] L. Caffarelli, B. Gidas and J. Spruck, Asymptotic symmetry and local behavior of semilinear elliptic equations with critical Sobolev growth, Comm. Pure Appl. Math., 42 (1989) pp. 271-297

[7] L. Caffarelli, H. Shahgholian and K. Yeressian, A minimization problem with free boundary related to a cooperative system, Duke Math. J., 167 (2018) pp. 1825-1882.

[8] L. Caffarelli and L. Silvestre, An extension problem related to the fractional Laplacian, Comm. Partial Differential Equations, 32 (2007) pp. 1245-1260.

[9] J. Case and S. A. Chang, On fractional GJMS operators, Comm. Pure Appl. Math., 69 (2016) pp. 1017-1061.

[10] W. X. Chen and C. Li, Classification of solutions of some nonlinear elliptic equations, Duke Math. J., 63 (1991) pp. 615-622.

[11] W. Chen and C. Li, B. Ou, Classification of solutions for an integral equation, Comm. Pure Appl. Math., 59 (2006) pp. 330343.

[12] Sun-Yung A. Chang and M. Gonzalez, Fractional Laplacian in conformal geometry, Advances in Mathematics, 226 (2011) pp. 1410-1432.

[13] M.G. Crandall and P.H. Rabinowitz, Some continuation and variation methods for positive solutions of nonlinear elliptic eigenvalue problems, Arch. Rat. Mech. Anal., 58 (1975) pp. 207-218.

[14] J. Davila, L. Dupaigne and J. Wei, On the fractional Lane-Emden equation, Trans. Amer. Math. Soc., 369 (2017) pp. 60876104.

[15] J. Davila, L. Dupaigne, K. Wang and J. Wei, A monotonicity formula and a Liouville-type theorem for a fourth order supercritical problem, Advances in Mathematics, 258 (2014) pp. 240-285.

[16] O. Druet and E. Hebey, Stability for strongly coupled critical elliptic systems in a fully inhomogeneous medium, Analysis and PDE, (2009) pp. 305-359.

[17] O. Druet, E. Hebey and J. Vétois, Bounded stability for strongly coupled critical elliptic systems below the geometric threshold of the conformal Laplacian, Journal of Functional Analysis, 258 (2010) pp. 999-1059.

[18] E. C. Evans, Partial regularity for stationary harmonic maps into spheres. Arch. Rational Mech. Anal., 116 (1991) pp. 101113.

[19] E. C. Evans, Monotonicity formulae for variational problems, Philos. Trans. R. Soc. Lond. Ser. A Math. Phys. Eng. Sci. 371 (2013), no. 2005, 9 pp.

[20] A. Farina; On the classification of solutions of the Lane-Emden equation on unbounded domains of $\mathbb{R}^{n}$, J. Math. Pures Appl., (9) 87 (2007) pp. 537-561.

[21] M. Fazly, Rigidity results for stable solutions of symmetric systems, Proc. Amer. Math. Soc., 143 (2015) pp. 5307-5321.

[22] M. Fazly and N. Ghoussoub, De Giorgi type results for elliptic systems, Calc. Var. Partial Differential Equations, 47 (2013) pp. 809-823.

[23] M. Fazly and H. Shahgholian, Regularity of free boundaries and partial regularity of weak solutions for coupled elliptic systems, In Progress.

[24] M. Fazly and J. Wei, On finite Morse index solutions of higher order fractional Lane-Emden equations, American Journal of Mathematics, 139 (2017) pp. 433-460.

[25] W. H. Fleming, On the oriented Plateau problem. Rend. Circ. Mat. Palermo, (2) 11 (1962) pp. 69-90

[26] B. Gidas and J. Spruck, A priori bounds for positive solutions of nonlinear elliptic equations, Comm. Partial Differential Equations, 6 (1981) pp. 883-901.

[27] B. Gidas, W. M. Ni and L. Nirenberg, Symmetry of positive solutions of nonlinear elliptic equations in $\mathbb{R}^{n}$, in Mathematical Analysis and Applications, Part A, Adv. in Math. Suppl. Stud., 7a, Academic Press, New York-London, 1981, 369-402.

[28] Y. Giga and R. V. Kohn, Asymptotically self-similar blow-up of semilinear heat equations, Comm. Pure Appl. Math., 38 (1985) pp. 297-319. 
[29] E. Hebey, Critical elliptic systems in potential form, Adv. Differential Equations, 11 (2006) pp. 511-600.

[30] E. Hebey and P. D. Thizy, Stationary Kirchhoff systems in closed high dimensional manifolds, Commun. Contemp. Math., 18,1550028 (2016) 53 pp.

[31] D. D. Joseph and T. S. Lundgren, Quasilinear Dirichlet problems driven by positive sources, Arch. Rational Mech. Anal., 49 (1972/73) pp. 241- 269.

[32] Y. Li, Remark on some conformally invariant integral equations: the method of moving spheres, J. Eur. Math. Soc. (JEMS), 6 (2004) pp. 153-180.

[33] C. S. Lin, A classification of solutions of a conformally invariant fourth order equation in $\mathbb{R}^{N}$, Comment. Math. Helv., 73 (1998) pp. 206-231.

[34] B. Noris, H. Tavares, S. Terracini and G. Verzini, Uniform Hölder bounds for nonlinear Schrödinger systems with strong competition, Comm. Pure Appl. Math., 63 (2010) pp. 267-302.

[35] F. Pacard, Partial regularity for weak solutions of a nonlinear elliptic equation, manuscripta mathematica, 79 (1993) pp. 161-172.

[36] A. Petrosyan, H. Shahgholian. and N. Uraltseva, Regularity of free boundaries in obstacle-type problems, Graduate Studies in Mathematics, 136. American Mathematical Society, Providence, RI, 2012. x+221.

[37] P. Price. A monotonicity formula for Yang-Mills fields, Manuscripta Math., 43 (1983) pp. 131-166.

[38] X. Ros-Oton and J. Serra, Local integration by parts and Pohozaev identities for higher order fractional Laplacians, Discrete Contin. Dyn. Syst. A, 35 (2015) pp. 2131-2150.

[39] R. Schoen, Analytic aspect of the harmonic map problem, Seminar on nonlinear partial differential equations (Berkeley, Calif., 1983), 321-358, Math. Sci. Res. Inst. Publ., 2, Springer, New York, 1984.

[40] R. Schoen and K. Uhlenbeck, $A$ regularity theory for harmonic maps, J. Differential Geometry, 17 (1982) pp. 307-335.

[41] S. Terracini, G. Verzini and A. Zilio, Uniform Hölder regularity with small exponent in competition-fractional diffusion systems, Discrete Contin. Dyn. Syst., 34 (2014) pp. 2669-2691.

[42] J. Wei and T. Weth, Radial solutions and phase separation in a system of two coupled schrödinger equations, Arch. Rational Mech. Anal. 190 (2008) pp. 83-106.

[43] J. Wei and X. Xu; Classification of solutions of higher order conformally invariant equations, Math. Ann., 313 (1999) pp. 207-228.

[44] G. S. Weiss, A homogeneity improvement approach to the obstacle problem, Invent. Math., 138 (1999) pp. 23-50.

[45] G. S. Weiss, Partial regularity for weak solutions of an elliptic free boundary problem, Communications in Partial Differential Equations 23 (1998) pp. 439-455.

[46] D. Yafaev, Sharp constants in the Hardy-Rellich inequalities, Journal of Functional Analysis, 168 (1999) pp. 121-144.

[47] R. Yang, On higher order extensions for the fractional Laplacian, preprint. http://arxiv.org/pdf/1302.4413v1.pdf 